\title{
Blood Pressure Variability and Therapeutic Implications in Hypertension and Cardiovascular Diseases
}

\author{
Chiara Nardin ${ }^{1}\left[\right.$ : Marcello Rattazzi ${ }^{1} \cdot$ Paolo Pauletto $^{2}$
}

Received: 7 May 2019 / Accepted: 31 August 2019 / Published online: 26 September 2019

(C) The Author(s) 2019

\begin{abstract}
Blood pressure (BP) is characterized by continuous dynamic and spontaneous oscillations occurring over lifetime and defining the so-called blood pressure variability (BPV). BPV has been associated with target organ damage, cardiovascular $(\mathrm{CV})$ risk and death, suggesting the use of BPV as a new target in hypertension management in addition to mean BP values lowering. The purpose of the review is to focus on the therapeutic implications of BPV and summarize the effects of different drug classes on various types of BPV. Despite most first-line antihypertensive medications contribute to reduce both short and long term BPV, calcium channel blockers (CCBs) as monotherapy or fixed-combination therapy appear to be the most effective on BPV control. Further randomized interventional trials are needed to investigate which drug combinations are most appropriate according to patient CV risk stratification, in order to improve their CV outcomes.
\end{abstract}

\section{Introduction}

Blood pressure variability (BPV) is defined by continuous dynamic and spontaneous fluctuations occurring over lifetime. BPV can be seen in very short-term (seconds or minutes, beat-to beat BPV), short-term (within a day, 24-h BPV), mid-term (between days, day-to-day BPV) and longterm (between clinic visits over months and years, visit-tovisit BPV). These oscillations are physiological and reflect the interplay of different mechanisms in response to internal and external stimuli, such as the cardiovascular (CV) control systems (beat-to-beat BPV), circadian rhythm (24-h BPV) and seasonal variations (visit-to-visit BPV). The detailed characteristics of the different type of BPV are summarized in Table 1 [1]. However, much is still unknown regarding the underlying factors [2]. Over the years, a growing number of clinical and observational studies have demonstrated an independent relationship between both short and long term $\mathrm{BPV}$ and the risk of CV events and death, regardless of mean blood pressure (BP) levels. Recent data from the Valsartan

Chiara Nardin

cnardin88@gmail.com

1 Department of Medicine-DIMED, Medicina Interna $\mathrm{I}^{\wedge}-\mathrm{Ca}^{\prime}$ Foncello Hospital, University of Padova, Via Ospedale, 31100 Treviso, Italy

2 O.R.A.S. SpA, Ospedale Riabilitativo di Alta Specializzazione, Motta Di Livenza, Italy
Antihypertensive Long-term Use Evaluation trial (VALUE), of approximately 14,000 hypertensive middle aged and older subjects, reported a 10\% increase in risk of death and a $15 \%$ increase in risk of $\mathrm{CV}$ events for $5 \mathrm{mmHg}$ increase in standard deviation (SD) of visit-to-visit and within-visit systolic $\mathrm{BPV}$, respectively [3]. Palatini and co-workers, focusing on 1206 stage 1 young hypertensives (mean age $33 \pm 8$ years), found that a 24-h higher systolic BP variability was associated with a greater number of fatal and non-fatal CV events during a median follow up of 15.4 years [4]. Indeed, changes in BPV have been associated with target organ damage such as arterial stiffness [5, 6], left ventricular hypertrophy [7], decline in renal function [8], subclinical brain small vessel disease [9] and the risk of developing foot ulcers in diabetes [10]. Therefore, although the current guidelines do not include the use of BPV as a target in hypertension management, controlling BPV should be considered as a new goal in addition to mean BP values lowering.

The current narrative review of the literature focuses on the therapeutic implications of BPV, summarizing the effects of different drug classes on various types of BPV and the role of lifestyle modifications, in order to improve the management of $\mathrm{CV}$ risk associated with hypertension. 
Table 1 Characteristics, indices of assessment and determinants of the different types of BPV

\begin{tabular}{|c|c|c|c|}
\hline Type of BPV & Methods for assessment & Main types of index & Determinants \\
\hline $\begin{array}{l}\text { Very short-term BPV (seconds or minutes, } \\
\text { beat-to beat) }\end{array}$ & Continuous BP recordings & $\begin{array}{l}\text { SD } \\
\text { CoV } \\
\text { ARV } \\
\text { Spectral analysis }\end{array}$ & $\begin{array}{l}\text { Neurohormonal factors (baroreceptor reflex, } \\
\text { sympathetic drive) } \\
\text { Emotion and stress }\end{array}$ \\
\hline Short-term BPV (within a day, 24-h) & $\begin{array}{l}\text { ABPM } \\
\text { HBPM }\end{array}$ & $\begin{array}{l}\text { SD } \\
\text { CoV } \\
\text { 24-h weighted SD } \\
\text { ARV } \\
\text { 24-h VIM } \\
\text { Spectral analysis }\end{array}$ & $\begin{array}{l}\text { Circadian rhythm } \\
\text { Nocturnal dipping of BP; night/day ratio } \\
\text { Obstructive sleep apnea } \\
\text { Neurohormonal factors (glucocorticoids, } \\
\text { RAAS system) } \\
\text { Emotional and behavioural factors }\end{array}$ \\
\hline Mid-term BPV (between days, day-to-day) & $\begin{array}{l}\text { ABPM over } 48 \mathrm{~h} \\
\text { HBPM }\end{array}$ & $\begin{array}{l}\text { SD } \\
\text { CoV } \\
\text { ARV } \\
\text { VIM }\end{array}$ & $\begin{array}{l}\text { Choice of antihypertensive treatment } \\
\text { Adherence to therapy } \\
\text { Vascular factors (endothelial damage, arterial } \\
\text { compliance) }\end{array}$ \\
\hline $\begin{array}{l}\text { Long-term BPV (between seasons, visit-to } \\
\text { visit) }\end{array}$ & $\begin{array}{l}\text { ABPM } \\
\text { HBPM } \\
\text { OBPM }\end{array}$ & $\begin{array}{l}\text { SD } \\
\text { CoV } \\
\text { ARV } \\
\text { VIM }\end{array}$ & $\begin{array}{l}\text { Choice of antihypertensive treatment } \\
\text { Adherence to therapy } \\
\text { Vascular factors (endothelial damage, arterial } \\
\text { compliance) } \\
\text { Seasonal changes }\end{array}$ \\
\hline
\end{tabular}

$B P V$ blood pressure variability, $S D$ standard deviation of $\mathrm{BV}$ values, $C o V$ coefficient of variation, assessed by dividing SD by the corresponding mean BP and multiplied by $100, A R V$ average real variability, the average of the absolute differences between consecutive BP measurement, VIM variability independent of the mean, $A B P M$ ambulatory blood pressure monitoring, $H B P M$ home blood pressure monitoring, $O B P M$ office blood pressure monitoring, $R A A S$ renin-angiotensin-aldosterone system

\section{Short Term Blood Pressure Variability and Therapeutic Implications}

\subsection{Circadian Blood Pressure Variability}

Given the influence of circadian rhythm on short-term BPV, some clinical studies have been mainly focused on the ability of drugs of restoring the normal 24-h pattern of BP, reducing morning surge and re-establishing the nocturnal dipping pattern (see Table 2). Therefore, drugs administration-time differences have been studied in order to normalize circadian BP profile and reduce short-term BPV. In the MAPEC study (Monitorización Ambulatoria para Predicción de Eventos Cardiovasculares, i.e., Ambulatory Blood Pressure Monitoring for Prediction of Cardiovascular Events), Hermida and colleagues, focusing on bedtime chronotherapy, hypothesised that the administration of $\geq 1$ antihypertensive drug at bedtime could be associated with a better BP control and a greater reduction in $\mathrm{CV}$ risk compared with conventional therapy. They found that subjects taking medications at bedtime exhibited a lower mean nocturnal BP and a lower CV risk after a median follow up of 5.6 years compared with those who ingest all drugs in the morning [11]. Other trials have demonstrated the beneficial effects of angiotensin receptor blockers (ARBs) bedtime intake on nocturnal BP dipping and within-day BPV $[12,13]$. Blocking the overnight increase in RAAS activity by nocturnal ingestion of ARBs and ACE (angiotensin-converting enzyme) inhibitors, regardless of their half-life, has been seen to be superior to any other treatment mechanism in reducing not only $\mathrm{CV}$ risk but also diabetes [14]. The sleep-time administration of calcium channel blockers (CCBs) as monotherapy or fixedcombination therapy has been also associated with decreased BPV and morning surge of BP, together with a better tolerance $[15,16]$. Relatively few studies have investigated the role of beta blockers (BBs) in bedtime chronotherapy. Acelajado and colleagues reported a better control of circadian BPV after nocturnal ingestion of nebivolol, although their cohort was very small $(\mathrm{N}=42)$ [17]. Despite these evidences, it should be specified that the bedtime chronotherapy may not be recommended as a general approach for all hypertensives, but it may be beneficial for subjects without nocturnal BP fall, in order to improve their BP control and to reduce circadian BPV. A personalised approach to individual BP pattern may represent the optimal strategy to manage CV risk in hypertensive patients.

\subsection{Non-circadian Blood Pressure Variability}

Evidences collected in the last years have suggested that most first-line antihypertensive medications contribute to reduce short term BPV. However, some differences between drug classes in the degree of beneficial effects have been reported (see Table 2).

Many trials compared the effects of CCBs, ARBs and diuretics on short term BPV. In the X-CELLENT Study (the Natrilix SR Versus Candesartan and Amlodipine in 


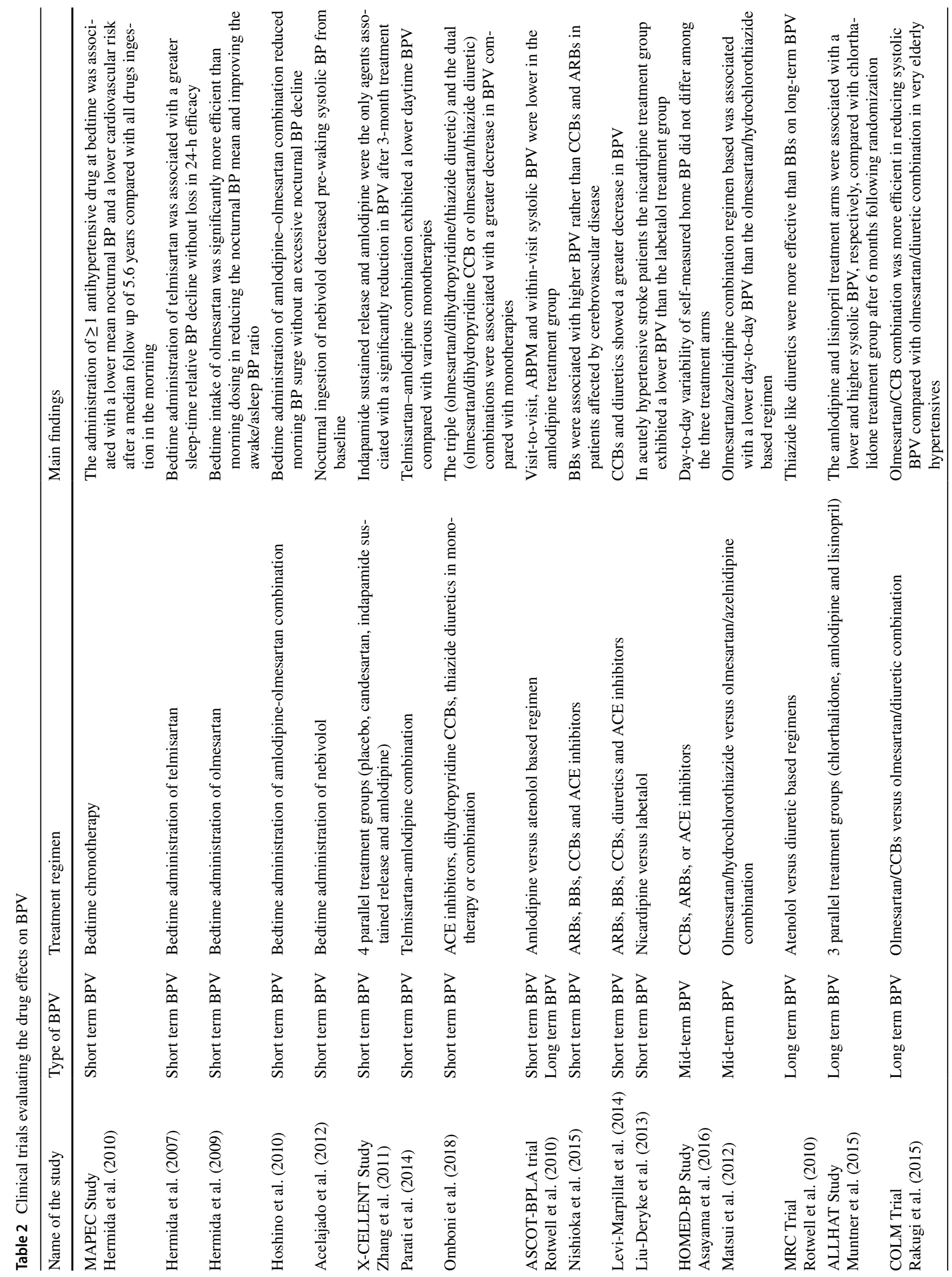


the Reduction of Systolic Blood Pressure in Hypertensive Patients) 577 middle-aged hypertensive subjects were recruited and treated according to 4 parallel treatment groups (placebo, candesartan, indapamide sustained release and amlodipine). The authors found that after 3 months of treatment, despite similar BP lowering effects, only amlodipine and indapamide sustained release were associated with the greater reduction in daytime, night-time and 24-h and daytime and $24 \mathrm{~h}$ systolic BPV, respectively. Interestingly, they also showed that the BPV lowering effect of amlodipine was associated with reduction of both BP and heart rate variability (HRV), whereas reduced HRV at night was the main alteration associated with indapamide [18]. In a large analysis of approximately 4000 adults the telmisartan/ amlodipine combination exhibited the lower daytime BPV [19], whereas another previous meta-analysis of approximately 5000 patients reported a greater BPV reduction in the telmisartan/amlodipine treatment arm compared with telmisartan/hydrochlorothiazide group [20]. Omboni and colleagues demonstrated that the triple combination olmesartan/dihydropyridine $\mathrm{CCB} /$ thiazide diuretic and the dual combinations (Olmesartan/dihydropyridine CCB or dihydropyridine $\mathrm{CCB} /$ thiazide diuretic) were associated with the larger decrease in BVP compared with placebo and monotherapies. The association was maintained, albeit weak, even after adjustment for treatment effect on mean BP, suggesting that an independent effect of these drugs on BPV may be present [21].

Of the relatively few studies investigating the effect of BBs on short term BPV, most demonstrated their inferiority on BPV reduction compared with the other antihypertensive drug classes. In the ASCOT-BPLA trial (Anglo-Scandinavian Cardiac Outcomes Trial-Blood Pressure Lowering Arm) daytime systolic BPV was lower in the amlodipinebased regimen than atenolol based regimen in a population of approximately 20,000 hypertensive subjects at high CV risk [22]. Nishioka and colleagues investigated a population of 309 patients with a history of cerebrovascular disease and found a higher BPV associated with BBs rather than CCBs or ARBs [23]. These findings have been also confirmed by other authors in subjects with essential hypertension [24] and stroke [25].

In summary, data from comparative clinical trials suggest that, although most first-line antihypertensive medications contribute to reduce short term BPV, treatment regimen containing CCBs are more favourable on BPV reduction than the other drug classes. This suggestion should be taken in account for the management of hypertensive patients, especially those at high $\mathrm{CV}$ risk or with $\mathrm{CV}$ diseases.

The effects of lifestyle modifications on short term BPV reduction have not been fully investigated. The largest study assessing the relationship between lifestyle and 24-h BPV has been published in 2017 by Maseli and colleagues [26] 
In their large cohort of approximately 2000 healthy subjects aged 25-41 years, they found an inverse correlation between healthy lifestyle and daytime, nighttime and weighted 24-h BPV. Interestingly this relationship, albeit attenuated, was maintained after adjustment for mean BP values. Endothelial dysfunction, baroreceptor reflex alterations and arterial stiffness, which are associated with unhealthy habits like smoking, could partly explain this correlation. However, it should be noted that the cohort was young, healthy and thus likely more sensitive to lifestyle modifications. Therefore, further researches are needed, in order to elucidate the effects of healthy lifestyle on short term BPV in hypertensive adults.

\section{Mid-term Blood Pressure Variability and Therapeutic Implications}

Day-to-day BPV finds its location halfway between short term BPV and long term BPV. Indeed, it may be influenced by mechanisms acting both over a short time, such as subject's activities, sleep wakefulness cycle and physiological factors, and over a long time, like climate variations and patient adherence. Despite its demonstrated association with target organ damage [27] and CV death [28], relatively few studies have investigated the impact of antihypertensive therapy on mid-term BPV (see Table 2). In the HOMEDBP Study (The multicenter Hypertension Objective Treatment Based on Measurement by Electrical Devices of Blood Pressure) 2484 middle-aged hypertensive participants were randomized to first-line treatment with CCB, ARB, or ACE inhibitor. After a median of 7.3 years following randomization, the authors found that only self-measured evening BPV predicted CV outcomes and day-to-day variability of selfmeasured home BP did not differ among the three treatment arms [29]. In The Japan Combined Treatment With Olmesartan and a Calcium-Channel Blocker Versus Olmesartan and Diuretics Randomized Efficacy Study, 207 hypertensive subjects were randomly allocated to treatment with hydrochlorothiazide or azelnidipine after 12 weeks of olmesartan monotherapy [30]. Despite similar systolic BP lowering between the two groups, a higher decrease in day-to-day $\mathrm{BPV}$ was associated with $\mathrm{CCB} / \mathrm{ARB}$ combination treatment compared with diuretic/ARB group during the follow up period. Interestingly, an independent relationship between changes in BPV and aortic pulse wave velocity, a marker of arterial stiffness, was found. This suggests that CCB's effect on BPV may be mediated not only from peripheral muscular arteries relaxation but also by an improvement of large arteries stiffness.

\section{Long Term Blood Pressure Variability and Therapeutic Implications}

Poor drug adherence has been hypothesised to be one of the most common factors affecting visit-to-visit BPV [31]; indeed, recent data reported that many patients (nearly 50\%) do not take all their medications [32]. Although the statistically significant association between non-adherence and increased visit-to-visit BPV does not fully explain the relationship between BPV and CV risk [33], improving drugadherence should be the first target for BPV reduction.

Growing evidences from post-hoc analyses of controlled clinical trials suggest that the effects of antihypertensive drugs on long-term BPV may contribute to reduce CV risk associated with hypertension (see Table 2). Webb and colleagues performed a metanalysis of 398 trials reviewing the effects of antihypertensive treatments on interindividual BPV, a surrogate of systolic visit-to-visit BPV, and risk of stroke. The metanalysis showed that CCBs and non-loop-diuretics decreased interindividual BPV, whereas ARBs, ACE inhibitors and BBs increased it. Particularly, compared with placebo, CCBs were the most effective in reducing interindividual BPV. This may partly explain the drug-class disparities on risk of stroke [34]. ASCOT-BPLA, MRC (Medical Research Council) and ALLHAT studies are the largest multicentre randomized controlled trials taken into account for retrospective analyses regarding the effects of different treatment arms on long term BPV. Data from ASCOT-BPLA study, of approximately 19,000 hypertensive subjects, showed an increased visit-to-visit BPV associated with atenolol compared with CCB based treatments, whereas in the MRC trial thiazide like diuretics were more effective than BBs on long-term BPV [22]. In the ALLHAT study 24,000 participants were randomized to chlortalidone, amlodipine or lisinopril. After 6-28 months following randomization, subjects in the amlodipine arm showed a 0.36 lower SD of systolic BP, whereas those treated with lisinopril exhibited a 0.77 higher SD of systolic BP compared with chlorthalidone treatment group [35]. The superiority of CCBs on reducing BPV compared with $\mathrm{ARB}, \mathrm{BB}$ or diuretic based regimens has been confirmed from other authors. In a sub-analysis of COLM trial (The Combination of OLMesartan), Rakugi and colleagues found that visit-to-visit systolic BPV was smaller in the olmesartan/CCBs treatment group compared with olmesartan/diuretic group, especially in very elderly Japanese hypertensives with isolated systolic hypertension [36]. In the COPE trial (The Combination Therapy of Hypertension to Prevent Cardiovascular Events), the benidipine/diuretic combination was more effective on long-term BPV than benidipine/ARBs and benidipine/BBs combinations [37]. However, conflicting data have been reported from other authors. In the ELSA trial (European 
Lacidipine Study on Atherosclerosis) no differences on visitto-visit BPV were found between BBs and CCBs in mildto-moderate hypertensive subjects [38]. Similar effects on seasonal BPV between the single-pill fixed-dose combination of $\mathrm{ARB} / \mathrm{CCB}$ and $\mathrm{ARB} /$ diuretic were also reported by Shiga and colleagues [39].

In summary, either as monotherapy or in combination, CCBs have been associated with the most effective long term BPV lowering. The mechanisms responsible for the beneficial effects of CCBs on BPV have not been completely clarified. Through their arterial vasodilatory effects, they might increase arterial compliance and improve baroreceptor function and autonomic nervous system regulation.

Among non-pharmacological interventions weight loss and salt reduction have not been associated with long term BPV lowering. 1820 subjects with high-normal diastolic BP were randomized to weight loss, salt reduction, their combination or usual care. The authors found no significant differences in visit-to-visit BPV between treatment groups after 36 months of follow up. However, some limitations should be cited: the cohort was relatively young and normotensive and systolic high-normal BP was not taken in account [40]. Thus, an independent effect of lifestyle modifications on long term BPV control cannot be excluded, suggesting the need of further researches.

\section{Conclusions}

Data available from literature demonstrate that reduce $\mathrm{BPV}$ may contribute to $\mathrm{CV}$ risk prevention, suggesting the use of BPV as a new target in hypertension management. Although most first-line antihypertensive drug classes contribute to attenuate BPV, long-acting CCBs appear to be the most effective treatment on BPV control. However, further randomized interventional trials are needed to investigate which drug combinations are most appropriate according to patient CV risk stratification. Given their role on short term BPV control, lifestyle modifications should also be taken in account as an effective strategy to ameliorate $\mathrm{CV}$ risk management in hypertensive patients.

The current guidelines do not include the use of BPV as a target in hypertension treatment, considering it as an optional index, probably because of its uncommon routine clinical use. This may be due to the absence of established threshold levels to discriminate pathological from physiological BPV, together with the lack of a standardized method of BPV assessment during daily clinical activity. These limitations lead to the need of further trials in order to make the BPV available and easy to use as a routine approach in daily clinical practice.

\section{Compliance with Ethical Standards}

Conflict of interest On behalf of all authors, the corresponding author states that there is no conflict of interest.

Ethical approval This article does not contain any studies with human participants or animals performed by any of the authors.

Open Access This article is distributed under the terms of the Creative Commons Attribution-NonCommercial 4.0 International License (http://creativecommons.org/licenses/by-nc/4.0/), which permits any noncommercial use, distribution, and reproduction in any medium, provided you give appropriate credit to the original author(s) and the source, provide a link to the Creative Commons license, and indicate if changes were made.

\section{References}

1. Parati G, Stergiou GS. Blood pressure variability: clinical relevance and application. J Clin Hypertens. 2018;20:1133-7.

2. Malik EZ, Abdulhadi B, Mezue KN, Lerma EV, Hon F, Rangaswami J. Clinical hypertension: blood pressure variability. Diseasea-Month. 2018;64:5-13.

3. Mehlum MH, Liestøl K, Kjeldsen SE, Julius S, Hua TA, Rothwell $\mathrm{PM}$, et al. Blood pressure variability and risk of cardiovascular events and death in patients with hypertension and different baseline risks. Eur Heart J. 2018;39(C):2243-51.

4. Palatini P, Saladini F, Mos L, Fania C, Mazzer A, Cozzio S, et al. Short-term blood pressure variability outweighs average 24-h blood pressure in the prediction of cardiovascular events in hypertension of the young. J Hypertens. 2019;37:1419-26.

5. Zhou TL, Henry RMA, Stehouwer CDA, Van Sloten TT, Reesink KD, Kroon AA. Blood pressure variability, arterial stiffness, and arterial remodeling: the Maastricht Study. Hypertension. 2018;72:1002-10.

6. Kim J, Park S, Yan P, Jeffers BW. Effect of inter-individual blood pressure variability on the progression of atherosclerosis in carotid and coronary arteries: a post hoc analysis of the NORMALISE and PREVENT studies. Eur Hear J Cardiovasc Pharmacother. 2017;3:82-9.

7. Mustafa EM, Strătoaie OI, Ușetescu ROM. Blood pressure variability and left ventricular mass in hypertensive patients. Curr Health Sci J. 2016;42(1):47-50.

8. Wang X. Twenty-four-hour systolic blood pressure variability and renal function decline in elderly male hypertensive patients with well-controlled blood pressure. Clin Interv Aging. 2018:13:533-40.

9. Filomena J, Riba-Llena I, Vinyoles E, Tovar JL, Mundet X, Castañé X, et al. Short-term blood pressure variability relates to the presence of subclinical brain small vessel disease in primary hypertension. Hypertension. 2015;66(3):634-40.

10. Palatini P. Risk of developing foot ulcers in diabetes. J Hypertens. 2018;36(11):2132-4.

11. Hermida RC, Ayala DE, Mojón A, Fernández JR. Influence of circadian time of hypertension treatment on cardiovascular risk: results of the MAPEC study. Chronobiol Int. 2010;27(8):1629-51.

12. Hermida RC, Ayala DE, Fernández JR, Calvo C. Comparison of the efficacy of morning versus evening administration of telmisartan in essential hypertension. Hypertension. 2007;50(4):715-22.

13. Hermida RC, Ayala DE, Chayán L, Mojón A, Fernández JR. Administration-time-dependent effects of olmesartan on the 
ambulatory blood pressure of essential hypertension patients. Chronobiol Int. 2009;26(1):61-79.

14. Hermida RC, Ayala DE, Fernández JR, Mojón A, Smolensky MH. Hypertension: new perspective on its definition and clinical management by bedtime therapy substantially reduces cardiovascular disease risk. Eur J Clin Investig. 2018;48:e12909.

15. Hoshino A, Nakamura $T$, Matsubara $H$. The bedtime administration ameliorates blood pressure variability and reduces urinary albumin excretion in amlodipine-olmesartan combination therapy. Clin Exp Hypertens. 2010;32(7):416-22.

16. Hermida RC, Ayala DE, Fernández JR, Mojón A, Smolensky $\mathrm{MH}$, Fabbian F, et al. Administration-time differences in effects of hypertension medications on ambulatory blood pressure regulation. Chronobiol Int. 2013;30:280-314.

17. Acelajado MC, Pisoni R, Dudenbostel T, Oparil S, Calhoun DA, Glasser SP. Both morning and evening dosing of nebivolol reduces trough mean blood pressure surge in hypertensive patients. J Am Soc Hypertens. 2012;6(1):66-72.

18. Zhang Y, Agnoletti D, Safar ME, Blacher J. Effect of antihypertensive agents on blood pressure variability: the natrilix SR versus candesartan and amlodipine in the reduction of systolic blood pressure in hypertensive patients (X-CELLENT) study. Hypertension. 2011;58(2):155-60.

19. Parati G, Dolan E, Ley L, Schumacher H. Impact of antihypertensive combination and monotreatments on blood pressure variability: assessment by old and new indices. Data from a large ambulatory blood pressure monitoring database. J Hypertens. 2014;32(6):1326-33.

20. Parati G, Schumacher H, Bilo G, Mancia G. Evaluating 24-h antihypertensive efficacy by the smoothness index: a meta-analysis of an ambulatory blood pressure monitoring database. J Hypertens. 2010;28(11):2177-83.

21. Omboni S, Kario K, Bakris G, Parati G. Effect of antihypertensive treatment on 24-h blood pressure variability: pooled individual data analysis of ambulatory blood pressure monitoring studies based on olmesartan mono or combination treatment. J Hypertens. 2018;36(4):720-33.

22. Rothwell PM, Howard SC, Dolan E, O’Brien E, Dobson JE, Dahlöf B, et al. Effects of $\beta$ blockers and calcium-channel blockers on within-individual variability in blood pressure and risk of stroke. Lancet Neurol. 2010;9(5):469-80.

23. Nishioka R, Kinoshita S, Shiibashi M, Shimazu T, Nakazato Y, Yamamoto T, et al. Evaluation of the differences in the effects of antihypertensive drugs on blood pressure variability by 24-hour ambulatory blood pressure monitoring in chronic cerebrovascular disease. J Stroke Cerebrovasc Dis. 2015;24(8):1848-54.

24. Levi-Marpillat N, MacQuin-Mavier I, Tropeano AI, Parati G, Maison P. Antihypertensive drug classes have different effects on short-term blood pressure variability in essential hypertension. Hypertens Res. 2014;37(6):585-90. https://doi.org/10.1038/ hr.2014.33.

25. Liu-Deryke X, Levy PD, Parker D, Coplin W, Rhoney DH. A prospective evaluation of labetalol versus nicardipine for blood pressure management in patients with acute stroke. Neurocrit Care. 2013;19(1):41-7.

26. Maseli A, Aeschbacher S, Schoen T, Fischer A, Jung M, Risch $\mathrm{M}$, et al. Healthy lifestyle and blood pressure variability in young adults. Am J Hypertens. 2017;30(7):690-9.

27. Matsui Y, Ishikawa J, Eguchi K, Shibasaki S, Shimada K, Kario K. Maximum value of home blood pressure: a novel indicator of target organ damage in hypertension. Hypertension. 2011;57(6):1087-93.

28. Kikuya M, Ohkubo T, Metoki H, Asayama K, Hara A, Obara $\mathrm{T}$, et al. Day-by-day variability of blood pressure and heart rate at home as a novel predictor of prognosis. Hypertension. 2008;52(6):1045-50.

29. Asayama K, Ohkubo T, Hanazawa T, Watabe D, Hosaka M, Satoh $\mathrm{M}$, et al. Does antihypertensive drug class affect day-to-day variability of self-measured home blood pressure? the HOMED-BP Study. J Am Heart Assoc. 2016;5(3):1-15.

30. Matsui Y, Kario K, Shimada K, Hoshide S, Ishikawa J, O'Rourke MF. Combined effect of angiotensin II receptor blocker and either a calcium channel blocker or diuretic on day-by-day variability of home blood pressure. Hypertension. 2012;59(6):1132-8.

31. Weber MA. Blood pressure variability and cardiovascular prognosis: implications for clinical practice. Eur Heart J. 2017;38(37):2823-6.

32. Azizi M, Pereira H, Hamdidouche I, Gosse P, Monge M, Bobrie $\mathrm{G}$, et al. Adherence to antihypertensive treatment and the blood pressure-lowering effects of renal denervation in the renal denervation for hypertension (DENERHTN) trial. Circulation. 2016;134(12):847-57.

33. Kronish IM, Lynch AI, Oparil S, Whittle J, Davis BR, Simpson $\mathrm{LM}$, et al. The association between antihypertensive medication nonadherence and visit-to-visit variability of blood pressure: findings from the antihypertensive and Lipid-Lowering Treatment to Prevent Heart Attack Trial (ALLHAT). Hypertension. 2017;68(1):39-45.

34. Webb AJ, Fischer U, Mehta Z, Rothwell PM. Effects of antihypertensive-drug class on interindividual variation in blood pressure and risk of stroke: a systematic review and meta-analysis. Lancet. 2010;375(9718):906-15.

35. Muntner P, Levitan EB, Lynch AI, Simpson LM, Whittle J, Davis $\mathrm{BR}$, et al. Effect of chlorthalidone, amlodipine and lisinopril on visit-to-visit variability of blood pressure: results from the Antihypertensive and Lipid-Lowering Treatment to Prevent Heart Attack. J Clin Hypertens. 2015;16(5):323-30.

36. Rakugi H, Ogihara T, Saruta T, Kawai T, Saito I, Teramukai S, et al. Preferable effects of olmesartan/calcium channel blocker to olmesartan/diuretic on blood pressure variability in very elderly hypertension. J Hypertens. 2015;33(10):2165-72.

37. Umemoto S, Ogihara T, Matsuzaki M, Rakugi H, Ohashi Y, Saruta T. Effects of calcium channel blocker-based combinations on intra-individual blood pressure variability: post hoc analysis of the COPE trial. Hypertens Res. 2015;39(1):46-53.

38. Mancia G, Facchetti R, Parati G, Zanchetti A. Visit-to-visit blood pressure variability in the European Lacidipine Study on Atherosclerosis. J Hypertens. 2012;30(6):1241-51.

39. Shiga Y, Miura S, Adachi S, Suematsu Y, Sugihara M. Visit-tovisit variability and seasonal variation in blood pressure with single-pill fixed-dose combinations of angiotensin II receptor blocker/calcium channel blocker and angiotensin II receptor blocker/diuretic in hypertensive patients. J Clin Med Res. 2015;7(10):802-6.

40. Diaz KM, Muntner P, Levitan EB, Brown MD, Babbitt DM, Shimbo D. The effects of weight loss and salt reduction on visitto-visit blood pressure variability: results from a multicenter randomized controlled trial. J Hypertens. 2014;32(4):840-8. 